Research Article

Human and Medical Genetics

\title{
ANRIL rs1333049 C/G polymorphism and coronary artery disease in a North Indian population - Gender and age specific associations
}

\author{
Naindeep $\operatorname{Kaur}^{1}$ (1) , Jagtar Singh ${ }^{1}$ (1) and Sreenivas Reddy ${ }^{2}$ (1) \\ ${ }^{1}$ Department of Biotechnology, Panjab University, Chandigarh, India. \\ ${ }^{2}$ Department of Cardiology, Postgraduate Institute of Medical Education and Research, Chandigarh, India.
}

\begin{abstract}
Many studies conducted worldwide substantiate a role of genetic polymorphisms in non-coding regions linked with coronary artery disease (CAD). One such single nucleotide polymorphism (SNP) of a non-coding RNA in the INK4 locus (ANRIL) i.e. rs $1333049 \mathrm{C} / \mathrm{G}$ in the vicinity of cell cycle regulating genes is documented to have a role in CAD risk. In this study we aimed to determine the association of ANRIL rs $1333049 \mathrm{C} / \mathrm{G}$ with CAD in a North Indian population. Five hundred disease free controls and $500 \mathrm{CAD}$ patients were genotyped using allele specific ARMS-PCR method. High risk association of rs 1333049 was seen in both heterozygous and mutant genotypes (OR=2.883, 95\% $\mathrm{Cl}=1.475-5.638$ and $\mathrm{p}=0.002$ and $\mathrm{OR}=6.717,95 \% \mathrm{Cl}=3.444-13.102$ and $\mathrm{p}<0.001$ respectively). Gender stratified analysis revealed risk association in both heterozygous and mutant genotypes in males. However, risk association in the mutant genotype and females was documented. Similarly, risk association was seen in subjects above 40 years of age in heterozygous and mutant genotypes. Similarly, risk association was reported in obese, sedentary lifestyle, positive family history and smoking in the heterozygous and mutant genotype and with diabetes in the mutant GG genotype. The study revealed high risk association of ANRIL rs1333049 with CAD and other risk factors.
\end{abstract}

Keywords: Genetic polymorphism, Coronary artery disease, North Indian Population, ARMS-PCR, epidemiology study.

Received: January 31, 2019; Accepted: December 15, 2019.

\section{Introduction}

Coronary artery disease (CAD) has become epidemic worldwide and a major barrier to sustainable human development. It has been lately observed that around 16.5 million people above 20 years of age in United States of America (U.S.) suffer from CAD. Not only that, the prevalence increases in both genders with a gradual increase in age (Sanchis-Gomar et al., 2016). The incidence in developing countries like India is also alarming and studies have reported a boost in CAD prevalence since past half century. There are a number of established key modifiable and non-modifiable factors like age, gender, genetics, smoking, dyslipidemia, hypertension, diabetes, obesity, high-fat diet, physical inactivity, drug abuse, alcohol consumption and mental stress attributing significant risk towards the disease.

An individual's risk of harboring CAD is inflected by the interplay between genetic and lifestyle factors established by the multifactorial nature of CAD. A genetic component in CAD is validated from the increased risk in first degree relatives of the affected individuals, high lifetime risk in the offspring if parents are affected and high concordance

Send correspondence to Sreenivas Reddy. Department of Cardiology, Postgraduate Institute of Medical Education and Research, 160012, Chandigarh, India. E-mail: reddycardio2911@gmail.com in monozygotic than dizygotic twins. The first Genome Wide Association (GWA) studies for CAD were published in 2007 and since then, a number of genetic variants at various chromosomal loci specific to CAD in various populations have been identified (Scheffold et al., 2011).

GWA studies document a locus on chromosome 9p21 linked to CAD (Jarinova et al., 2009; Cunnington et al., 2010; Ahmed et al., 2013). Though this $58 \mathrm{~kb}$ locus lacks the genes associated with atherosclerosis, an antisense non-coding RNA in the INK4 locus (ANRIL) gene dwells within the vicinity of cell cycle regulating genes in this region. It is reported to be in strong linkage disequilibrium with cell cycle proliferatory genes such as cyclin dependant kinase inhibitors $2 A$ and $2 B$ (CDKN2A and $C D K N 2 B$ ) (Cunnington et al., 2010). $C D K N 2 A$ is basically a tumor suppressor gene and encodes two proteins viz. p14ARF and p16. p16 controls the G1 to $\mathrm{S}$ transition in the cell cycle and p14ARF stimulates cell cycle arrest in G2 phase, subsequently leading to cell death. $C D K N 2 B$ lies adjoining the $C D K N 2 A$ and encodes proteins that inhibit the cell cycle G1 progression (Cunnington et al., 2010).

$C D K N 2 B$ anti-sense RNA (CDKN2B-AS1) spans about $126.3 \mathrm{~kb}$ and overlaps with $C D K N 2 B(p 15)$ at the 5 ' end and comprises of 20 exons that are prone to alternative splicing (Jarinova et al., 2009) and reported to be linked to CAD risk (Matsuoka et al., 2015; Dehghan et al., 2016), hypertension 
(Bayoglu et al., 2016) and stroke (Bai et al., 2014). CDKN2B-AS or CDKN2B-AS1 or INK4 are used as synonyms for ANRIL. The ANRIL locus is reported to alter the expression of neighbouring genes by apparently acting either by chromatin remodeling, DNA methylation, gene silencing or RNA interference (Jarinova et al., 2009).

The SNP rs1333049 C/G is positioned in the 3'UTR (untranslated region) of $C D K N 2 B-A S 1$ and considered to have a crucial role in advancement of cardio and cerebrovascular disease by modifying dynamics of vascular smooth muscle cell proliferation (Cunnington et al., 2010). The Wellcome Trust Case Control consortium study has documented rs1333049 as displaying powerful association with CAD (Consortium, 2007). The association of rs 1333049 was studied in CAD (Dechamethakun et al., 2014; Haslacher et al., 2016), atherosclerosis (Bochenek et al., 2013) and Alzheimer's disease (Popov et al., 2010).

The present study was conducted with the aim of determining allelic and genotypic frequencies of ANRIL rs1333049 and risk association with CAD and other selected parameters in a North Indian population.

\section{Material and Methods}

\section{Study population}

One thousand individuals aged 25-70 years of both sexes were enrolled to evaluate the role of LOXI rs 11053646 $\mathrm{G} / \mathrm{C}$ and rs $1050283 \mathrm{C} / \mathrm{T}$ polymorphisms in CAD. Five hundred patients belonging to North Indian states (Jammu and Kashmir, Haryana, Chandigarh, Punjab, Himachal Pradesh, New Delhi, Uttaranchal, Uttar Pradesh, Uttarakhand and Rajasthan) visiting the Department of Cardiology at Postgraduate Institute of Medical Education and Research, Chandigarh and documented CAD on coronary angiogram with more than $50 \%$ stenosis in at least one epicardial coronary artery) were registered as cases. Subjects with acute/chronic infection, hepatic dysfunction, renal dysfunction, severe heart failure, hypo or hyperthyroidism, pregnancy and malignancy were excluded. Five hundred healthy individuals satisfying the inclusion criteria (with absence of any cardiac disorder, chronic diseases such as diabetes, hypertension, hypo- or hyperthyroidism, tuberculosis, hepatitis, AIDS, malignancy and pregnancy) were enrolled as controls. Subjects with history of smoking, alcohol consumption and tobacco chewing were also excluded. Majority of the controls were donors at the blood donation camps. A written informed consent was given by all participants prior to enrollment. The study was approved by the Institutional Ethics Committee, Panjab University, Chandigarh, India and performed according to the "Ethical Guidelines for Biomedical Research on Human Participants, 2006" as proposed by the Indian Council of Medical Research and Ministry of Health, Govt. of India.

\section{Biometric and biochemical measurements}

Anthropometric parameters like height, weight, waist to hip ratio, BMI and blood pressure were noted. Risk factors for CAD like diabetes, hypertension, dyslipidemia, family history, smoking and drinking habits were recorded. Lipid profile, fasting blood glucose, hsCRP, uric acid Apolipoprotein A1 and Apolipoprotein B determination was done by standard biochemical methods.

\section{DNA isolation, SNP selection and genotyping}

Five milliliters of venous blood sample was collected in EDTA-coated vials and DNA was isolated by the sodium saline citrate buffer method (Roe et al., 1996). Genotyping of the ANRIL rs1333049 C/G polymorphism was done by allele-specific ARMS-PCR using sequence specific primers (forward primer for $\mathrm{C}$ allele: TCC TCA TAC TAA CCA TAT GAT CAA CAG TTC, forward primer for $\mathrm{G}$ allele: TCC TCA TAC TAA CCA TAT GAT CAA CAG TTG, internal control primer sequence: GAA GAT CAT ACC CGA AGT AGA GCT GC. For all forward primers a common reverse primer was used, with the sequence ATA CCA CAG TGA ACA TAA TTG TGC ATA CAT). The PCR was carried out in a thermal cycler with a total volume of $25 \mu \mathrm{L}$ containing: 10X PCR Buffer, $3 \mathrm{mM} \mathrm{MgCl} 2,1 \mathrm{mg} / \mathrm{mL}$ nuclease free BSA, 50 pmol each of allele specific forward primer, reverse primer and internal control primer, $10 \mathrm{mM}$ of each dNTP, $0.125 \mathrm{U}$ Taq polymerase and $2 \mu$ genomic DNA. The PCR cycle included an initial denaturation step of $5 \mathrm{~min}$ at $94{ }^{\circ} \mathrm{C}$, followed by 35 cycles with denaturation for $30 \mathrm{~s}$ at 94 ${ }^{\circ} \mathrm{C}$, annealing for $30 \mathrm{~s}$ at $59{ }^{\circ} \mathrm{C}$ elongation for $30 \mathrm{~s}$ at $72{ }^{\circ} \mathrm{C}$ and a final elongation of $10 \mathrm{~min}$ at $72^{\circ} \mathrm{C}$.

Separate PCR was performed for both alleles of one SNP. The DNA fragments obtained were separated on 3\% agarose gel stained with $\mathrm{EtBr}$ followed by visualization with UV transilluminator. A $280 \mathrm{bp}$ fragment signified CC or GG genotype and $500 \mathrm{bp}$ signified the internal control. If bands were seen for both alleles, it was interpreted as the heterozygous genotype (Figure 1). Correctness of genotyping was checked by re-genotyping of $10 \%$ of the samples. Results from the repeated samples were $100 \%$ consistent with our primary results.

\section{Statistical analysis}

Continuous variables that were not normally distributed were expressed as the mean \pm standard deviation (SD). Categorical variables were reported as counts and percentages. Chi-square test was used to calculate the difference between baseline characteristics. To investigate the association of SNP and the susceptibility to CAD, multivariate logistic regression was applied adjusting for age and gender. Furthermore, recessive and dominant models were analysed. Stratified analysis for gender and age was also done for the assessment of association and expressed in odds ratio (OR) and $95 \%$ confidence interval (CI). A statistical significance of $p<0.05$ was considered for the analysis. All the data was analysed using SPSS version 20.0 (SPSS, Inc., Chicago, IL) and Epi Info version 3.4.7 (CDC, Atlanta, GA). 


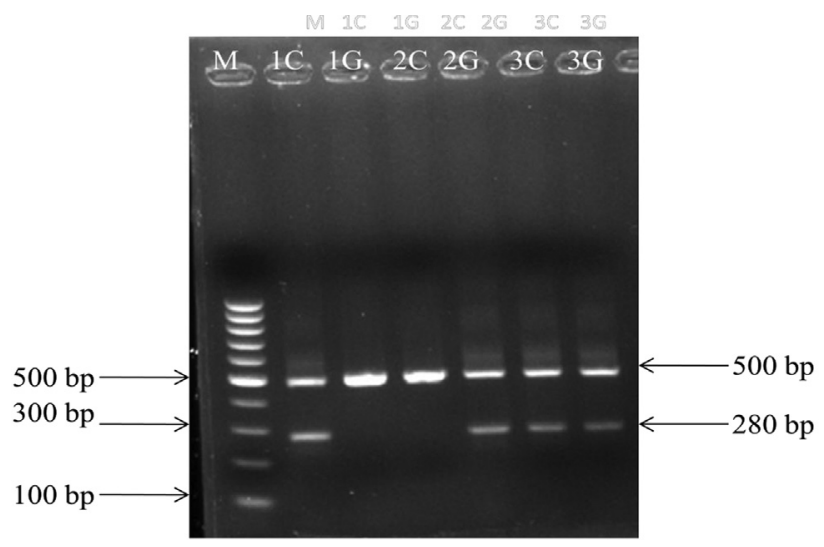

Figure 1 - Allele specific ARMS-PCR products of ANRIL rs 1333049 C/G polymorphism on 3\% agarose gel. Lane M: 100 bp ladder, Lane 1C and 1G: homozygous wild CC genotype (500 and $280 \mathrm{bp}$ ), Lane $2 \mathrm{C}$ and $2 \mathrm{G}$ : homozygous mutant GG genotype (500 and $280 \mathrm{bp}$ ), Lane 3C and 3G: heterozygous CG genotype (500 and $280 \mathrm{bp}$ )

\section{Results}

The distribution of allele frequencies of the selected polymorphism followed the Hardy-Weinberg equilibrium. The baseline parameters of patients and controls are listed in Table 1 . The results revealed a statistically significant variation between the two groups with respect to age, gender, smoking, drinking, waist to hip ratio, lifestyle, family history, dyslipidemia, diabetes, diet, hypertension, occupation, exercise, fasting blood sugar, uric acid, TC, VLDL, LDL, Apo A1, Apo B, but not with total lipids, triglycerides, BMI, HDL and hsCRP.

Results revealed that the mutant $(\mathrm{G})$ allele was more prevalent in patients (35.4\%) than controls (33.8\%) exhibiting a non-significant association to $\mathrm{CAD}$ with $\mathrm{OR}=0.930$, 95\% $\mathrm{CI}=0.77-1.13$ and $\mathrm{p}=0.451$ (Table 2 ).

The genotypic frequencies revealed the wild (CC) genotype to have a higher frequency in control $(49.2 \%)$ than in CAD patients (33.2\%). The heterozygous genotype (CG) was found to be highly prevalent among the cases $(62.8 \%)$ in comparison to the controls $(34.0 \%)$ with $\mathrm{OR}=2.883,95 \%$ $\mathrm{CI}=1.475-5.638, \mathrm{p}=0.002$ and the homozygous mutant $(\mathrm{GG})$ genotype had a higher frequency in controls $(16.8 \%)$ than in the cases $(4.0 \%)$ thereby conferring an increased risk with a high significance $\mathrm{p}<0.001, \mathrm{OR}=6.717$ and $95 \% \mathrm{CI}=3.444$ 13.102 (Table 2). The dominant and recessive models were also analysed to see the association of the polymorphism with CAD. A protective association was seen in the dominant model $(\mathrm{OR}=0.582,95 \% \mathrm{CI}=0.402-0.842$ and $\mathrm{p}=0.004)$ whereas elevated risk association with CAD was observed in the recessive model $(\mathrm{OR}=4.609,95 \% \mathrm{CI}=2.431-8.741$ and $\mathrm{p}$ $<0.001)$.

The data was stratified on the basis of age i.e. below 40 years and above 40 years (Table 3 ). High risk association in subjects above 40 years was documented for both the heterozygous and the mutant genotypes, with $\mathrm{OR}=2.647,95 \%$ $\mathrm{CI}=1.287-5.447$ and $\mathrm{p}=0.008$ and $\mathrm{OR}=5.506,95 \%$ $\mathrm{CI}=2.688-11.278$ and $\mathrm{p}<0.001$ respectively. Also the $\mathrm{G}$ allele showed risk association $(\mathrm{OR}=1.600,95 \% \mathrm{CI}=1.226$ -
Table 1 - Baseline characteristics of the studied population.

\begin{tabular}{|c|c|c|c|}
\hline Phenotypic traits & Controls n $(\%)$ & Cases n $(\%)$ & $\mathrm{p}$ \\
\hline Age (Mean \pm SD; years) & $50.95 \pm 10.18$ & $56.08 \pm 9.55$ & $<0.001 *$ \\
\hline Waist to hip ratio & $0.91 \pm 0.08$ & $0.97 \pm 0.16$ & $0.001^{*}$ \\
\hline \multicolumn{4}{|l|}{ Blood Pressure } \\
\hline \multicolumn{4}{|l|}{$\mathrm{SBP}(\mathrm{mmHg})$} \\
\hline & $121.13 \pm 9.92$ & $133.67 \pm 15.25$ & $0.001^{*}$ \\
\hline \multicolumn{4}{|l|}{ DBP (mmHg) } \\
\hline & $80.35 \pm 7.07$ & $90.81 \pm 13.45$ & $0.046^{*}$ \\
\hline Gender & & & $0.043^{*}$ \\
\hline Males & $370(74)$ & $397(79.4)$ & \\
\hline Females & $130(26)$ & $103(20.6)$ & \\
\hline BMI & & & 0.069 \\
\hline Normal & $270(54)$ & $296(59.2)$ & \\
\hline Obese & $230(46)$ & $204(40.8)$ & \\
\hline Smoking status & Nil & & $<0.001^{*}$ \\
\hline Non-smoker & $327(65.4)$ & & \\
\hline Smoker & $173(34.6)$ & & \\
\hline Drinking status & Nil & & $<0.001^{*}$ \\
\hline Non-Drinker & $348(69.6)$ & & \\
\hline Drinker & $152(30.4)$ & & \\
\hline Address & & & 0.342 \\
\hline Rural & $233(46.6)$ & $248(49.6)$ & \\
\hline Urban & $267(53.4)$ & $252(50.4)$ & \\
\hline Family history & & & $<0.001^{*}$ \\
\hline Nil & $47(89.4)$ & $304(60.8)$ & \\
\hline$+\mathrm{ve}$ & $53(10.6)$ & $196(39.2)$ & \\
\hline Lifestyle & & & $<0.001^{*}$ \\
\hline Active & $431(86.2)$ & $352(70.4)$ & \\
\hline Sedentary & $69(13.8)$ & $148(29.6)$ & \\
\hline Diabetes & Nil & & $<0.001^{*}$ \\
\hline Negative & $353(70.6)$ & & \\
\hline Positive & $147(29.4)$ & & \\
\hline Hypercholesterolemia & Nil & $500(100)$ & $<0.001^{*}$ \\
\hline \multicolumn{4}{|l|}{ Negative } \\
\hline \multicolumn{4}{|l|}{ Positive } \\
\hline Hypertension & Nil & $500(100)$ & $<0.001^{*}$ \\
\hline \multicolumn{4}{|l|}{ Negative } \\
\hline \multicolumn{4}{|l|}{ Positive } \\
\hline Diet & & & $0.001 *$ \\
\hline Veg & $425(85)$ & $385(77)$ & \\
\hline Non veg & $75(15)$ & $115(23)$ & \\
\hline CK MB & $54.46 \pm 45.81$ & $34.36 \pm 41.81$ & $<0.001^{*}$ \\
\hline APO A1 & $144.56 \pm 26.41$ & $121.09 \pm 42.36$ & $<0.001^{*}$ \\
\hline APO B & $98.67 \pm 36.54$ & $65.94 \pm 23.77$ & $<0.001^{*}$ \\
\hline hsCRP & $4.56 \pm 0.20$ & $2.35 \pm 0.22$ & $<0.001 *$ \\
\hline HDL-C & $52.35 \pm 3.12$ & $88.06 \pm 9.11$ & 0.673 \\
\hline LDL-C & $135.65 \pm 22.33$ & $76.54 \pm 32.50$ & $0.005^{*}$ \\
\hline VLDL-C & $48.57 \pm 21.08$ & $34.83 \pm 20.74$ & $0.043 *$ \\
\hline FBG & $82.73 \pm 19.30$ & $111.56 \pm 39.59$ & $0.016^{*}$ \\
\hline URIC ACID & $9.80 \pm 3.45$ & $6.26 \pm 5.10$ & $0.006^{*}$ \\
\hline TC & $275.76 \pm 53.49$ & $144.95 \pm 37.22$ & $0.045^{*}$ \\
\hline TRIGLYCERIDES & $198.23 \pm 82.45$ & $145.37 \pm 65.62$ & 0.468 \\
\hline TL & $512.18 \pm 116.05$ & $436.05 \pm 112.36$ & 0.353 \\
\hline
\end{tabular}

BMI: body mass index, SBP: systolic blood pressure, DBP: diastolic blood pressure, hsCRP: high-sensitivity C-reactive protein (hs-CRP), TC: total cholesterol, TG: triglycerides, HDL-C: high-density lipoprotein cholesterol, LDL-C: low density lipoprotein cholesterol; VLDL-C: very low density lipoprotein, FBG: fasting blood glucose, TL: total lipids. ${ }^{*}=$ significant 
Table 2 - Genotypic and allelic frequencies of ANRIL rs 1333049 C/G polymorphism in controls and cases.

\begin{tabular}{|c|c|c|c|c|}
\hline \multirow[t]{2}{*}{ Genotypes } & \multirow{2}{*}{$\begin{array}{c}\text { Controls } \\
500(\%)\end{array}$} & \multirow{2}{*}{$\begin{array}{c}\text { Cases } \\
500(\%)\end{array}$} & \multicolumn{2}{|c|}{ Multiple logistic regression analysis } \\
\hline & & & $\mathrm{p}$ & OR $(95 \% \mathrm{CI})$ \\
\hline \multicolumn{5}{|c|}{ ANRIL $\mathrm{rs} 1333049 \mathrm{C} / \mathrm{G}$} \\
\hline $\mathrm{CC}$ & $246(49.2)$ & $166(33.2)$ & (ref.) & \\
\hline CG & $170(34)$ & $314(62.8)$ & $0.002 *$ & $2.883(1.475-5.638)$ \\
\hline GG & $84(16.8)$ & $20(4)$ & $<0.001 *$ & $6.717(3.444-13.102)$ \\
\hline Alleles C & $662(66.2)$ & $646(64.6)$ & (ref.) & \\
\hline G & $338(33.8)$ & $354(35.4)$ & 0.451 & $0.930(0.77-1.13)$ \\
\hline \multicolumn{5}{|c|}{ Dominant Model } \\
\hline $\mathrm{CC}$ & $246(49.2)$ & $166(33.2)$ & (ref.) & \\
\hline $\mathrm{CG}+\mathrm{GG}$ & $254(50.8)$ & $334(66.8)$ & $0.004 *$ & $0.582(0.402-0.842)$ \\
\hline \multicolumn{5}{|c|}{ Recessive Model } \\
\hline $\mathrm{CG}+\mathrm{CC}$ & $416(83.2)$ & $480(96)$ & (ref.) & \\
\hline GG & $84(16.8)$ & $20(4)$ & $<0.001 *$ & $4.609(2.431-8.741)$ \\
\hline
\end{tabular}

ref. $=$ reference; $\mathrm{OR}=$ odds ratio; $\mathrm{CI}=$ confidence interval; $\%=$ frequency; $*=$ significant

2.088 and $\mathrm{p}<0.001)$. For the age group less than 40 years, there is no mutant GG genotype in the cases. Therefore, calculations regarding allelic frequencies were not possible because one value is entirely missing. Nevertheless, a nonsignificant association could be seen in subjects below 40 years of age with the selected polymorphism. Stratification of the data on the basis of gender was also done (Table 3). In males, risk association was seen for both the heterozygous and the mutant genotypes, with $\mathrm{OR}=2.683,95 \% \mathrm{CI}=1.193$ 6.034 and $\mathrm{p}=0.017$ and $\mathrm{OR}=5.902,95 \% \mathrm{CI}=2.628-13.255$ and $\mathrm{p}<0.001$ respectively, whereas for females, only the mutant GG genotype revealed risk association, with $\mathrm{OR}=9.248,95 \% \mathrm{CI}=2.666-32.077$ and a highly significant $\mathrm{p}$ $<0.001$. Obesity also showed high risk association in heterozygous state with $\mathrm{OR}=3.546,95 \% \mathrm{CI}=1.157-10.863$ and $\mathrm{p}=0.027$ and mutant genotype with $\mathrm{OR}=8.633,95 \%$ $\mathrm{CI}=2.823-26.400$ and $\mathrm{p}<0.001$ (Table 3 ). Our study also intended to look for the association of sedentary lifestyle as a risk factor for CAD. Highly significant risk association was seen in $\mathrm{CG}$ and $\mathrm{GG}$ genotypes with $\mathrm{OR}=2.913,95 \%$ $\mathrm{CI}=1.390-6.105$ and $\mathrm{p}=0.005$ and $\mathrm{OR}=7.024, \quad 95 \%$ $\mathrm{CI}=3.358-14.693$ and $\mathrm{p}<0.001$ (Table 3). Positive family history also highlighted association with CAD risk with $\mathrm{OR}=5.805,95 \% \mathrm{CI}=0.373-8.726$ and $\mathrm{p}=0.018$ for the heterozygous genotype and $\mathrm{OR}=7.453,95 \% \mathrm{CI}=1.445-38.438$ and $\mathrm{p}=0.016$ for the mutant genotype (Table 3 ). No association of drinking with the polymorphism and CAD risk was seen. Nevertheless, strong risk association with both the hetero and mutant genotype was seen with smoking $(\mathrm{OR}=1.837, \quad 95 \% \quad \mathrm{CI}=0.548-2.279$ and $\mathrm{p}=0.011$ and $\mathrm{OR}=2.092,95 \% \mathrm{CI}=0.377-3.161$ and $\mathrm{p}=0.027$ respectively). However, only the mutant GG genotype showed risk association with diabetes with $\mathrm{OR}=2.330,95 \% \mathrm{CI}=1.458-3.857$ and $\mathrm{p}=0.006$ (Table 4).

\section{Discussion}

This study aimed to understand risk association of ANRIL rs $1333049 \mathrm{C} / \mathrm{G}$ to CAD in a North Indian population. Sequence specific ARMS-PCR was used for genotyping and results showed a considerable risk association towards CAD and the same was observed in the recessive model (Table 1). Moreover, the allelic frequencies also conferred a significant association with CAD ( $\mathrm{p}<0.05)$. Also, the rs $1333049 \mathrm{C} / \mathrm{G}$ polymorphism showed risk towards CAD for age above 40 years, males and females, obesity, sedentary lifestyle, family history, diabetes and smoking.

Numerous studies document ANRIL rs1333049 C/G polymorphism and its correlation with CAD risk and progression (Samani et al., 2007, 2008; Welcome Trust Case Control Consortium, 2007; Schunkert et al., 2008; Karvanen et al., 2009; Muendlein et al., 2009; Bressler et al., 2010; Dandona et al., 2010; Ellis et al., 2010; McPherson, 2010; Palomaki et al., 2010; Wang et al., 2010; Mendonça et al., 2011; O'Donnell et al., 2011; Angelakopoulou et al., 2012; Plichart et al., 2012) and ischaemic stroke (Karvanen et al., 2009; Smith et al., 2009). The above mentioned studies majorly comprised of Caucasian descent populations.

A few studies from India have tried to explore genetic polymorphisms at this selected locus. A GWA study done with a South Indian population on 9p21 locus reported two SNPs (rs2383207 and rs10757278) conferring elevated risk to CAD (AshokKumar et al., 2011). Also, the work done by Kumar et al. (2011) on the North Indian population, reports three SNPs (rs2383206, rs1333040 and rs10116277) at 9p21 locus to be associated with CAD risk. The rs 10757278 polymorphism at the same locus also correlates with CAD risk as reported by two studies by (Maitra et al., 2008; Bhanushali et al., 2011). Only two studies report data on $\mathrm{rs} 1333049 \mathrm{C} / \mathrm{G}$ and CAD risk in West and North Indian populations. (Bhanushali, et al., 2013) recruited 229 CAD patients and 136 controls from West India and revealed an association towards $\mathrm{CAD}$ with an $\mathrm{OR}=2.460,95 \% \mathrm{CI}=1.139-5.314$ and 
Table 3 - Stratified analysis for different parameters and its association with ANRIL rs 1333049 C/G polymorphism.

\begin{tabular}{|c|c|c|c|c|c|c|c|c|}
\hline \multirow[b]{2}{*}{ Genotype } & \multicolumn{4}{|c|}{ AGE $<40$ YEARS } & \multicolumn{4}{|c|}{ AGE $\geq 40$ YEARS } \\
\hline & Controls $379(\%)$ & Cases $27(\%)$ & $\mathrm{p}$ & OR $(95 \% \mathrm{CI})$ & Controls $121(\%)$ & Cases $473(\%)$ & $\mathrm{p}$ & $\mathrm{OR}(95 \% \mathrm{CI})$ \\
\hline $\mathrm{CC}$ & 193(50.9) & $8(29.6)$ & (ref.) & & $53(43.8)$ & $158(33.4)$ & (ref.) & \\
\hline $\mathrm{CG}$ & $121(31.9)$ & $19(70.4)$ & & $\begin{array}{l}\text { Can't b } \\
\text { calculated }\end{array}$ & $49(40.5)$ & $295(62.4)$ & $0.008^{*}$ & $\begin{array}{c}2.647 \\
(1.287-5.447)\end{array}$ \\
\hline GG & $65(17.2)$ & $0(0)$ & & $\begin{array}{l}\text { Can't b } \\
\text { calculated }\end{array}$ & $19(15.7)$ & $20(4.2)$ & $<0.001^{*}$ & $\begin{array}{c}5.506 \\
(2.688-11.278)\end{array}$ \\
\hline Allele C & $507(66.8)$ & $35(64.8)$ & (ref.) & & $155(64.5)$ & $611(64.3)$ & (ref.) & \\
\hline \multirow[t]{3}{*}{ Allele G } & $251(33.2)$ & $19(35.2)$ & 0.751 & $\begin{array}{c}0.912 \\
(0.511-1.626)\end{array}$ & $136(56.5)$ & $335(35.7)$ & $<0.001 *$ & $\begin{array}{c}1.600 \\
(1.226-2.088)\end{array}$ \\
\hline & \multicolumn{4}{|c|}{ MALE } & \multicolumn{4}{|c|}{ FEMALE } \\
\hline & Controls $370(\%)$ & Cases $397(\%)$ & $\mathrm{p}$ & OR $(95 \% \mathrm{CI})$ & Controls $130(\%)$ & Cases $103(\%)$ & $\mathrm{p}$ & OR $(95 \% \mathrm{CI})$ \\
\hline $\mathrm{CC}$ & $173(46.8)$ & $135(34)$ & (ref.) & & $73(56.2)$ & $31(30.1)$ & (ref.) & \\
\hline $\mathrm{CG}$ & $135(36.5)$ & $246(62)$ & $0.017^{*}$ & $\begin{array}{c}2.683 \\
(1.193-6.034)\end{array}$ & $35(26.9)$ & $68(66)$ & 0.062 & $\begin{array}{c}3.312 \\
(0.944-11.622)\end{array}$ \\
\hline GG & $62(16.8)$ & $16(4)$ & $<0.001^{*}$ & $\begin{array}{c}5.902 \\
(2.628-13.255)\end{array}$ & $22(16.9)$ & $4(3.9)$ & $<0.001^{*}$ & $\begin{array}{c}9.248 \\
(2.666-32.077)\end{array}$ \\
\hline Allele C & $481(65)$ & $516(65.3)$ & (ref.) & & 181(69.6) & $130(61.9)$ & (ref.) & \\
\hline \multirow[t]{3}{*}{ Allele G } & $259(35)$ & $278(34.7)$ & 0.862 & $\begin{array}{c}0.983 \\
(0.797-1.212)\end{array}$ & $79(30.4)$ & $76(38.1)$ & 0.138 & $\begin{array}{c}0.746 \\
(0.506-1.099)\end{array}$ \\
\hline & \multicolumn{4}{|c|}{ NON-OBESE } & \multicolumn{4}{|c|}{ OBESE } \\
\hline & Controls $270(\%)$ & Cases $296(\%)$ & $\mathrm{p}$ & OR $(95 \% \mathrm{CI})$ & Controls $230(\%)$ & Cases $204(\%)$ & $\mathrm{p}$ & OR $(95 \% \mathrm{CI})$ \\
\hline $\mathrm{CC}$ & $133(49.3)$ & $96(32.4)$ & (ref.) & & $113(49.1)$ & $70(34.3)$ & (ref.) & \\
\hline CG & $92(34.1)$ & $186(62.8)$ & 0.064 & $\begin{array}{c}2.681 \\
(1.136-6.327)\end{array}$ & $78(33.9)$ & $128(62.7)$ & $0.027^{*}$ & $\begin{array}{c}3.546 \\
(1.157-10.863)\end{array}$ \\
\hline GG & $45(16.7)$ & $14(4.7)$ & 0.076 & $\begin{array}{c}6.086 \\
(2.590-14.304)\end{array}$ & $39(17)$ & $6(2.9)$ & $<0.001 *$ & $\begin{array}{c}8.633 \\
(2.823-26.400)\end{array}$ \\
\hline Allele C & $358(66.2)$ & $378(64)$ & (ref.) & & $304(66)$ & $268(65.3)$ & & \\
\hline \multirow[t]{2}{*}{ Allele G } & $182(33.8)$ & 214(36) & 0.389 & $\begin{array}{c}0.641 \\
(0.390-1.053)\end{array}$ & $156(44)$ & $140(34.7)$ & 0.887 & $\begin{array}{c}1.432 \\
(0.603-3.401)\end{array}$ \\
\hline & \multicolumn{4}{|c|}{ ACTIVE LIFESTYLE } & \multicolumn{4}{|c|}{ SEDENTARY LIFESTYLE } \\
\hline Genotypes & Controls $69(\%)$ & Cases $148(\%)$ & $\mathrm{p}$ & $\mathrm{OR}(95 \% \mathrm{CI})$ & Controls $431(\%)$ & Cases $352(\%)$ & $\mathrm{p}$ & $\mathrm{OR}(95 \% \mathrm{CI})$ \\
\hline $\mathrm{CC}$ & $27(39.1)$ & $50(33.8)$ & (ref.) & & $219(50.8)$ & $116(33)$ & (ref.) & \\
\hline $\mathrm{CG}$ & $32(46.4)$ & $94(63.5)$ & 0.374 & $\begin{array}{c}2.129 \\
(0.402-11.274)\end{array}$ & $138(32)$ & $220(62.5)$ & $0.005^{*}$ & $\begin{array}{c}2.913 \\
(1.390-6.105)\end{array}$ \\
\hline GG & $10(14.5)$ & $4(2.7)$ & 0.099 & $\begin{array}{c}3.952 \\
(0.772-20.423)\end{array}$ & $74(17.2)$ & $16(4.5)$ & $<0.001 *$ & $\begin{array}{c}7.024 \\
(3.358-14.693)\end{array}$ \\
\hline Allele C & $86(61.4)$ & 194(64.6) & (ref.) & & $576(66.9)$ & $452(64.5)$ & (ref.) & \\
\hline \multirow[t]{3}{*}{ Allele G } & $52(38.6)$ & $102(35.4)$ & 0.511 & $\begin{array}{c}1.987 \\
(0.750-5.267)\end{array}$ & $286(33.1)$ & $252(35.5)$ & 0.277 & $\begin{array}{c}0.458 \\
(0.080-2.603)\end{array}$ \\
\hline & \multicolumn{4}{|c|}{ No Family History } & \multicolumn{4}{|c|}{ Family History } \\
\hline & Controls $447(\%)$ & Cases $304(\%)$ & $\mathrm{p}$ & $\mathrm{OR}(95 \% \mathrm{CI})$ & Controls $53(\%)$ & Cases $196(\%)$ & $\mathrm{p}$ & $\mathrm{OR}(95 \% \mathrm{CI})$ \\
\hline $\mathrm{CC}$ & $213(47.7)$ & $96(31.6)$ & (ref.) & & $33(62.3)$ & $70(35.7)$ & (ref.) & \\
\hline $\mathrm{CG}$ & $159(35.6)$ & $195(64.1)$ & 0.069 & $\begin{array}{c}2.830 \\
(1.299-6.163)\end{array}$ & $11(20.8)$ & $119(60.7)$ & $0.018^{*}$ & $\begin{array}{c}5.805 \\
(0.373-8.726)\end{array}$ \\
\hline GG & $75(16.8)$ & $13(4.3)$ & 0.055 & $\begin{array}{c}6.502 \\
(3.013-14.029)\end{array}$ & $9(17)$ & $7(3.6)$ & $0.016^{*}$ & $\begin{array}{c}7.453 \\
(1.445-38.438)\end{array}$ \\
\hline Allele C & $585(65)$ & $387(63.4)$ & (ref.) & & $77(70)$ & $259(66.4)$ & (ref.) & \\
\hline Allele G & $309(35)$ & $221(36.6)$ & 0.479 & $\begin{array}{c}0.925 \\
(0.745-1.147)\end{array}$ & $29(30)$ & $133(33.6)$ & 0.200 & $\begin{array}{c}0.733 \\
(0.455-1.179)\end{array}$ \\
\hline
\end{tabular}

$\mathrm{p}=0.022)$. Kashyap et al. (2018) in their study on North Indian population reported risk for both the allelic and genotypic frequencies. This study also showed an association with $\mathrm{CAD}$ with an $\mathrm{OR}=6.717,95 \% \mathrm{CI}=3.444-13.102$ and $\mathrm{p}$ $<0.001$. Thus, the results point towards the fact that both the North Indians as well as the West Indians are susceptible to CAD due to this polymorphic change in ANRIL rs1333049.
The multiple conventional risk factors for CAD such as diabetes, hypertension, dyslipidemia, etc. become additive with increasing age, thereby contributing to atherosclerosis leading to CAD. A positive risk association in the subjects above 40 years of age was seen in the study with the mutant genotype having an $\mathrm{OR}=5.506$ with a highly significant $\mathrm{p}<0.001$ (Table 2). However, Bhanushali et al. (2013) reported the SNP to be robustly associated with premature or 
Table 4 - Association of drinking, smoking and diabetes with ANRIL rs 1333049 C/G polymorphism.

\begin{tabular}{|c|c|c|c|c|}
\hline Genotype & Non-Drinker 348 (\%) & Drinker $152(\%)$ & p & OR $(95 \% \mathrm{CI})$ \\
\hline $\mathrm{CC}$ & $118(33.9)$ & $48(31.6)$ & (ref.) & \\
\hline $\mathrm{CG}$ & $216(62.1)$ & $98(64.5)$ & 0.708 & $0.926(0.615-1.394)$ \\
\hline GG & 14(4) & $6(3.9)$ & 0.740 & $1.20(0.414-3.477)$ \\
\hline Allele C & $452(21.8)$ & $194(63.8)$ & (ref.) & \\
\hline \multirow[t]{2}{*}{ Allele G } & $244(78.2)$ & $110(36.2)$ & 0.740 & $0.768(0.453-1.300)$ \\
\hline & Non-Smoker 327 (\%) & Smoker 173 (\%) & $\mathbf{p}$ & OR $(95 \%$ CI) \\
\hline $\mathrm{CC}$ & $111(33.9)$ & $55(31.8)$ & (ref.) & \\
\hline CG & $202(61.8)$ & $112(64.7)$ & $0.011^{*}$ & $1.837(0.548-2.279)$ \\
\hline GG & $14(4.3)$ & $6(3.5)$ & $0.027^{*}$ & $2.092(0.377-3.161)$ \\
\hline Allele C & $424(64.8)$ & $222(64.2)$ & (ref.) & \\
\hline \multirow[t]{2}{*}{ Allele G } & $230(35.2)$ & $124(35.8)$ & 0.841 & $1.124(0.333-3.798)$ \\
\hline & Non-diabetic 353 (\%) & Diabetic 147 (\%) & $\mathbf{p}$ & OR $(95 \%$ CI) \\
\hline $\mathrm{CC}$ & $115(32.6)$ & $51(34.7)$ & (ref.) & \\
\hline CG & $223(63.2)$ & $91(61.9)$ & 0.689 & $1.086(0.721-1.637)$ \\
\hline GG & $15(4.2)$ & $5(3.4)$ & $0.006^{*}$ & $2.330(1.458-3.857)$ \\
\hline Allele C & $453(64.1)$ & $193(65.6)$ & (ref.) & \\
\hline Allele G & $253(35.6)$ & $101(34.4)$ & 0.654 & $1.067(0.921-2.211)$ \\
\hline
\end{tabular}

the early onset CAD which is also supported by the results of Meng et al. (2008), Ellis et al. (2010) and the association was supported by meta-analysis study done by Palomaki et al. (2010). But in present study, no mutant in the CAD patients was found below 40 years of age in the pre-specified subgroup analysis based on age. The overall frequency of GG mutant genotype in our selected population is $10.4 \%$ i.e. only 104 individuals have the GG genotype thereby pointing towards its low prevalence in our selected North Indian population.

The discrepancy in results emphasizes the need to genotype all the risk variants particularly at this locus, as this will help in delineating the varied risk associations in different populations to CAD. However, the impact of the polymorphism with disease extent and severity is disputable with Ye et al. (2008) and Dandona et al. (2010) stating it as a predictor of severity, whereas Anderson et al. (2008) and Chen et al. (2009) contradicting it. Additionally, the present study results showed a strong association with the family history which is in accordance with previous work (Preuss et al., 2010; Scheffold et al., 2011). Gender stratified analysis depicted significant association in both the genders which is in harmony to the results reported by Ahmed et al. (2013) on Northern Pakistani population.

In summary, we conclude that ANRIL rs $1333049 \mathrm{C} / \mathrm{G}$ is associated with susceptibility to CAD in North Indian population and also associations with many risk factors have been documented. Although 9p21 locus association with risk of CAD is very well recognized, relationship with the clinical outcomes remains unclear and unanswered. The chosen SNP is intronically located but still can affect gene expression. Therefore, future studies with higher sample size, multiple SNPs from the locus and linkage studies are needed to authenticate our results that might cause identification of more SNPs at this particular locus as biomarkers for CAD predisposition.
Analyzing the SNPs which are substantially associated with CAD in North Indian population will be useful to identify promising SNP-CAD associations unique to the population. Moreover, CAD poses threat not only to an individual and his family but also to the community and the nation on the whole as the most productive years of one's life is spent struggling with the disease. The drastic change in lifestyle and eating habits and the increased tendency to rely on machines and other forms of assistance has substantially decreased one's physical effort and rendered individuals highly susceptible to CAD. Comprehending the genetic foundation of CAD is highly needed these days that will help in screening individuals at high risk and will also lay the groundwork for the coacervation of genetic data and routine clinical practice, which can one day spearhead the arena of "personalized medicine".

\section{Acknowledgments}

All the authors are thankful to the participants who consented to be a part of the study.

\section{Conflict of Interest}

None

\section{Author Contributions}

NK collected the samples, processed, genotyped and statistically analyzed all the data, JS provided all the financial support for carrying out the work, SR provided the control and patient blood samples. All the authors contributed equally in conceptualization, design analysis, planning, conduct, data analysis and manuscript preparation.

\section{References}

Ahmed W, Ali IS, Riaz M, Younas A, Sadeque A, Niazi AK, Niazi SH, Ali SHB, Azam M and Qamar R (2013) Associa- 
tion of ANRIL polymorphism (rs1333049: C > G) with myocardial infarction and its pharmacogenomic role in hypercholesterolemia. Gene 515:416-420.

Anderson JL, Horne BD, Kolek MJ, Muhlestein JB, Mower CP, Park JJ, May HT, Camp NJ and Carlquist JF (2008) Genetic variation at the $9 \mathrm{p} 21$ locus predicts angiographic coronary artery disease prevalence but not extent and has clinical utility. Am Heart J 156:e1152.

Angelakopoulou A, Shah T, Sofat R, Shah S, Berry DJ, Cooper J, Palmen J, Tzoulaki I, Wong A, Jefferis BJ, et al. (2012) Comparative analysis of genome-wide association studies signals for lipids, diabetes, and coronary heart disease: Cardiovascular Biomarker Genetics Collaboration. Eur Heart J 33:393-407.

AshokKumar M, Emmanuel C, Dhandapany PS, Rani DS, SaiBabu R, Cherian KM and Thangaraj K (2011) Haplotypes on 9 p21 modify the risk for coronary artery disease among Indians. DNA Cell Biol 30:105-110.

Bai Y, Nie S, Jiang G, Zhou Y, Zhou M, Zhao Y, Li S, Wang F, Lv Q, Huang Y et al. (2014) Regulation of CARD8 expression by ANRIL and association of CARD8 single nucleotide polymorphism rs2043211 (p. C10X) with ischemic stroke. Stroke 45:383-388.

Bayoglu B, Yuksel H, Cakmak HA, Dirican A and Cengiz M (2016) Polymorphisms in the long non-coding RNA CDKN2B-AS1 may contribute to higher systolic blood pressure levels in hypertensive patients. Clin Biochem 49:821-827.

Bhanushali AA, Parmar N, Contractor A, Shah VT and Das BR (2011) Variant on 9p21 is strongly associated with coronary artery disease but lacks association with myocardial infarction and disease severity in a population in Western India. Arch Med Res 42:469-474.

Bhanushali AA, Contractor A and Das BR (2013) Variant at 9p21 rs 1333049 is associated with age of onset of coronary artery disease in a Western Indian population: a case control association study. Genet Res (Camb) 95:138-145.

Bochenek G, Häsler R, El Mokhtari NE, König IR, Loos BG, Jepsen S, Rosensyiel P, Schreiber S and Schaefer AS (2013) The large non-coding RNA ANRIL, which is associated with atherosclerosis, periodontitis and several forms of cancer, regulates ADIPOR1, VAMP3 and C11ORF10. Hum Mol Genet 22:4516-4527.

Bressler J, Folsom AR, Couper DJ, Volcik KA and Boerwinkle E (2010) Genetic variants identified in a European genomewide association study that were found to predict incident coronary heart disease in the atherosclerosis risk in communities study. Am J Epidemiol 171:14-23.

Chen SN, Ballantyne CM, Gotto AM and Marian AJ (2009) The 9 p21 susceptibility locus for coronary artery disease and the severity of coronary atherosclerosis. BMC Cardiovasc Disord 9:3.

Cunnington MS, Santibanez Koref M, Mayosi BM, Burn J and Keavney B (2010) Chromosome 9p21 SNPs associated with multiple disease phenotypes correlate with ANRIL expression. PLoS Genet 6: e1000899.

Dandona S, Stewart AF, Chen L, Williams K, So D, O'Brien E, Glover C, Lemay M, Assogba O, Vo L et al. (2010) Gene dosage of the common variant 9p21 predicts severity of coronary artery disease. J Am Coll Cardiol 56:479-486.

Dechamethakun S, Ikeda S, Arai T, Sato N, Sawabe M and Muramatsu M (2014) Associations between the
CDKN2A/B, ADTRP and PDGFD polymorphisms and the development of coronary atherosclerosis in Japanese patients. J Atheroscler Thromb 21:680-690.

Dehghan A, Bis JC, White CC, Smith AV, Morrison AC, Cupples LA, Trompet S, Chasman DI, Lumley T, Volker U et al. (2016) Genome-wide association study for incident myocardial infarction and coronary heart disease in prospective cohort studies: the CHARGE consortium. PLoS One 11: e0144997.

Ellis KL, Pilbrow AP, Frampton CM, Doughty RN, Whalley GA, Ellis CJ, Palmer BR, Skelton L, Yandle TG, Palmer SC et al. (2010) A common variant at chromosome 9p21.3 is associated with age of onset of coronary disease but not subsequent mortality. Circ Cardiovasc Genet 3:286-293.

Haslacher H, Perkmann T, Ratzinger F, Grimm G, Exner M, Keller A, Schmetterer K, Priemer C, Endler G, Wagner O et al. (2016) 9p21.3 risk locus is associated with first-ever myocardial infarction in an Austrian cohort. J Cardiovasc Med (Hagerstown) 17:595-600.

Jarinova O, Stewart AF, Roberts R, Wells G, Lau P, Naing T, Wells G, Lau P, Naing T, Buerki C et al. (2009) Functional analysis of the chromosome $9 \mathrm{p} 21.3$ coronary artery disease risk locus. Arterioscler Thromb Vasc Biol 29:1671-1677.

Karvanen J, Silander K, Kee F, Tiret L, Salomaa V, Kuulasmaa K, Wiklund PG, Virtamo J, Sararela O, Perret C et al. (2009) The impact of newly identified loci on coronary heart disease, stroke and total mortality in the MORGAM prospective cohorts. Genet Epidemiol 33:237-246.

Kashyap S, Kumar S, Agarwal V, Misra DP, Rai MK and Kapoor A (2018) The association of polymorphic variants, rs2267788, rs 1333049 and rs2383207 with coronary artery disease, its severity and presentation in North Indian population. Gene 648:89-96.

Kumar J, Yumnam S, Basu T, Ghosh A, Garg G, Karthikeyan G and Sengupta S (2011) Association of polymorphisms in 9p21 region with CAD in North Indian population: replication of SNPs identified through GWAS. Clin Genet 79:588593.

Maitra A, Shanker J, Dash D, John S, Sannappa PR, Rao VS, Ramanna JK and Kakkar VV (2008) Polymorphisms in the IL6 gene in Asian Indian families with premature coronary artery disease - the Indian Atherosclerosis Research Study. Thromb Haemost 99:944-950.

Matsuoka R, Abe S, Tokoro F, Arai M, Noda T, Watanabe S, Horibe H, Fujimaki T, Oguri M, Kato K et al. (2015) Association of six genetic variants with myocardial infarction. Int $\mathrm{J}$ Mol Med 35: 1451-1459.

McPherson R (2010) Chromosome 9p21 and coronary artery disease. N Engl J Med 362:1736-1737.

Mendonça I, dos Reis RP, Pereira A, Café H, Serrão M, Sousa AC, Freitas AI, Guerra G, Freitas S, Freitas C, Ornelas I et al. (2011). Independent association of the variant rs1333049 at the 9p21 locus and coronary heart disease. Rev Port Cardiol 30:575-591.

Meng W, Hughes AE, Patterson CC, Belton C, Kee F and McKeown PP (2008) Chromosome 9p21.3 is associated with early-onset coronary heart disease in the Irish population. Dis Markers 25:81-85.

Muendlein A, Saely CH, Rhomberg S, Sonderegger G, Loacker S, Rein P, Beer S, Vonbank A, Winder T and Drexel H (2009) Evaluation of the association of genetic variants on the chromosomal loci 9p21.3, 6q25.1, and 2q36.3 with angio- 
graphically characterized coronary artery disease. Atherosclerosis 205:174-180.

O'Donnell CJ, Kavousi M, Smith AV, Kardia SL, Feitosa MF, Hwang SJ, Sun YV, Province MA, Aspelund T, Dehghan A et al. (2011) Genome-wide association study for coronary artery calcification with follow-up in myocardial infarction. Circulation 124:2855.

Palomaki GE, Melillo S and Bradley LA (2010) Association between 9p21 genomic markers and heart disease: a metaanalysis. JAMA 303:648-656.

Plichart M, Empana JP, Lambert JC, Amouyel P, Tiret L, Letenneur L, Berr C, Tzourio C and Ducimetière P (2012) Single polymorphism nucleotide rs 1333049 on chromosome 9p21 is associated with carotid plaques but not with common carotid intima-media thickness in older adults. A combined analysis of the Three-City and the EVA studies. Atherosclerosis 222:187-190.

Popov N and Gil J (2010) Epigenetic regulation of the INK4bARF-INK4a locus: in sickness and in health. Epigenetics 5:685-690

Preuss M, König IR, Thompson JR, Erdmann J, Absher D, Assimes TL, Blankenberg S, Boerwinkle E, Chen L, Cupples LA et al. (2010) Design of the Coronary ARtery DIsease Genome-Wide Replication And Meta-Analysis (CARDIoGRAM) Study: A Genome-wide association meta-analysis involving more than 22000 cases and 60000 controls. Circ Cardiovasc Genet 3:475-483.

Roe BA, Crabtree JS and Khan AS (1996) DNA isolation and sequencing. Wiley-Blackwell, vol. 11, 176 p.

Samani NJ, Erdmann J, Hall AS, Hengstenberg C, Mangino M, Mayer B, Dixon RJ, Meitinger T, Braund P, Wichmann HE et al. (2007) Genomewide association analysis of coronary artery disease. N Engl J Med 357:443-453.

Samani NJ and Schunkert H (2008) Chromosome 9p21 and cardiovascular disease: the story unfolds. Circ Cardiovasc Genet 1:81-84.
Sanchis-Gomar F, Perez-Quilis C, Leischik R and Lucia A (2016) Epidemiology of coronary heart disease and acute coronary syndrome. Ann Transl Med 4:256.

Scheffold T, Kullmann S, Huge A, Binner P, Ochs HR, Schöls W, Thale J, Motz W, Hegge FJ, Stellbrink C et at. (2011) Six sequence variants on chromosome 9p21.3 are associated with a positive family history of myocardial infarction: a multicenter registry. BMC Cardiovasc Disord 11:9.

Schunkert H, Götz A, Braund P, McGinnis R, Tregouet DA, Mangino M, Linsel-Nitschke P, Cambien F, Hengstenberg C, Stark K et al. (2008) Repeated replication and a prospective meta-analysis of the association between chromosome 9p21.3 and coronary artery disease. Circulation 117: 16751684.

Smith JG, Melander O, Lövkvist H, Hedblad B, Engström G, Nilsson P, Carlson J, Berglund G, Norrving B and Lindgren A (2009) Common genetic variants on chromosome 9p21 confers risk of ischemic stroke: a large-scale genetic association study. Circ Cardiovasc Genet 2:159-164.

Wang W, Peng W, Zhang X, Lu L, Zhang R, Zhang Q, Wang L, Chen Q and Shen W (2010) Chromosome 9p21.3 polymorphism in a Chinese Han population is associated with angiographic coronary plaque progression in non-diabetic but not in type 2 diabetic patients. Cardiovasc Diabetol 9:33.

Welcome Trust Case Control Consortium (2007) Genome-wide association study of 14,000 cases of seven common diseases and 3,000 shared controls. Nature 447:661-678.

Ye S, Willeit J, Kronenberg F, Xu Q and Kiechl S (2008) Association of genetic variation on chromosome $9 \mathrm{p} 21$ with susceptibility and progression of atherosclerosis: a populationbased, prospective study. J Am Coll Cardiol 52(5):378-384.

Associate Editor: Maria Cortez

License information: This is an open-access article distributed under the terms of the Creative Commons Attribution License (type CC-BY), which permits unrestricted use, distribution and reproduction in any medium, provided the original article is properly cited. 